\title{
Diagnostic Imaging Ordering Practices: Physician Perspectives and Implications for Decision Support
}

Janessa K. Griffith, Elizabeth M. Borycki and Andre W. Kushniruk

\begin{abstract}
This study explored how referring physicians order diagnostic imaging (DI) services, and possible methods to reduce inappropriate ordering. Telephone interviews were conducted with non-radiologist physicians (general practitioners and specialists). Interview data were analyzed using grounded theory.

Both appropriate and inappropriate DI ordering practices emerged as the overarching themes. Specifically, the majority of participants described their top methods of obtaining information support as (1) contacting another physician or (2) consulting the literature. Additionally, participants discussed contributing factors and solutions to inappropriate DI ordering, including clinical decision support systems. These results were used to inform the design of a DI decision support system prototype.

This study explored ways to reduce inappropriate DI ordering and identified socio-technical factors that need to be considered when developing ways to mitigate this phenomenon. Promoting more appropriate ordering can improve patient safety and the responsible use of limited diagnostic imaging resources.
\end{abstract}

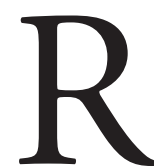

esearch in diagnostic imaging (DI) ordering practices can help to promote patient safety and the responsible use of limited healthcare resources by reducing unnecessary DI examinations. While research relating to why physicians may order inappropriately is present in the literature, how physicians order DI remains unclear.
Using key informant interviews, this study aimed to explore this knowledge gap. From the interview data, ordering (both appropriate and inappropriate) emerged as the overarching themes. This included several subthemes: what methods of information support a physician would seek during a challenging clinical scenario, and contributing factors and solutions to inappropriate ordering. These findings included the physician perspective on how information technology (IT; such as clinical decision support [CDS] systems) could be used to reduce inappropriate DI ordering. Findings were compared and contrasted to the current literature surrounding DI. With recent increases in DI use, examining ways to reduce unnecessary examinations is crucial in order to promote patient safety and the responsible use of DI resources.

\section{Background}

\section{Diagnostic Imaging}

For the purpose of this study, diagnostic imaging refers to radiography, computed tomography (CT), magnetic resonance imaging (MRI), sonography, nuclear medicine and positron emission tomography (PET). This study focused on the diagnostic features of these technologies, excluding DI used for screening or image-guided procedures.

\section{Increases in Use}

Examining physician DI ordering practices has become critical to understanding the underlying reasons for increases in DI use 
over the past decade. Specifically, in Canada between the fiscal years of 2003/4 and 2011/12, CT use increased from 2,767,849 to $4,377,919(58 \%)$ and MRI use increased from 768,302 to 1,718,633 (124\%) (Canadian Institute for Health Information [CIHI] 2013). These escalating figures warrant further examination of DI ordering practices.

\section{"Specifically, in Canada between the fiscal years of 2003/4 and 2011/12, CT use increased from 2,767,849 to 4,377,919 $(58 \%)$ and $\mathrm{MRI}$ use increased from 768,302 to $1,718,633$ (124\%) (CIHI 2013)."}

\section{Inappropriate Ordering}

According to the Canadian Association of Radiologists (CAR), a useful DI investigation "is one in which the result - positive or negative - will alter clinical management and/or add confidence to the physician's diagnosis" (2005a: 8). Meanwhile an inappropriate DI examination refers to several cases. First, unnecessary duplicate orders are considered inappropriate. For example, a patient might be ordered an MRI by the family physician and then again by a specialist physician (not knowing that the examination had already been ordered). As well, a case where the wrong DI modality is selected is also deemed to be an inappropriate order. For example, a physician might order a CT scan for a patient in a case where MRI would produce the same results. Here, the patient would be unnecessarily exposed to radiation; thus, the examination is considered inappropriate. A DI examination that does not contribute to patient management is also inappropriate. An example is when the treatment plan is unaffected regardless of whether the DI results are positive or negative. Similarly, performing examinations too early (e.g., before a cancer has progressed enough to be seen on the DI examination) is also inappropriate (CAR 2005a). Research surrounding DI ordering practices is critical as CAR suggests that up to $30 \%$ of diagnostic imaging in Canada could be inappropriate (CAR 2005b, 2010).

\section{Literature Review}

\section{Health Informatics Solutions}

CDS systems could potentially help to reduce inappropriate DI ordering, according to many researchers (Bautista et al. 2009; CAR 2010; Czembirek et al. 2002; Kahn and Tjahjono 1999; Sistrom 2005; Sistrom and Honeyman 2002). CDS systems can take various forms including clinical pathway diagrams and question formats (Government of Western Australia 2007; Ontario Ministry of Health and Long-Term Care 2009). As well, some can be included in a computerized order entry system or electronic health record (EHR), such as one used at the Massachusetts General Hospital (MGH n.d.). Different methods of reasoning can be used in CDS systems, such as artificial neural networks, Bayesian principles or rule-based techniques (Musen et al. 2006). CDS systems used in healthcare can improve the efficiency of tasks, facilitate learning and increase patient safety. Overall, CDS systems are used to support users in their decision-making processes (Health Council of Canada [HCC] 2010). In fact, the CDS system for DI used at MGH bent the utilization curve from a $12 \%$ annual increase for CT and MRI examinations to $1 \%$ and $7 \%$, respectively (Rosenthal et al. 2006).

As well, several pilot studies have been conducted in Canadian settings (Atlantic Health Sciences Centre et al. 2005; CAR 2007; Government of Saskatchewan 2007). However, none of these studies described how physicians reach a decision or what methods of decision support are sought when faced with a challenging clinical scenario. Thus, more research is needed to determine the effectiveness of CDS systems as means to promote more appropriate DI ordering practices.

Although research relating to $w h y$ physicians inappropriately order is present in the literature, how physicians order is still unclear; this is a key piece to better understanding DI ordering practices. In other words, human factors have only been discussed after a system has been implemented. By considering user requirements, this a priori research can inform the development of CDS systems, or other IT, for optimal user acceptance.

The current literature also suggests other solutions for reducing inappropriate DI ordering, including improving access and awareness of guidelines, educating physicians and medical students and using technology such as CDS systems. First, improving access and awareness is important for guideline acceptance and adoption by referring physicians - especially in such a quickly changing field (Bautista et al. 2009). This could be achieved by having guidelines available through the most popularly reported sources of information support used: Google Internet searches, specialty journals, continuing medical education, meetings and "UptoDate (an evidence-based, peerreviewed medical information resource)" (Bautista et al. 2009: 1581). Therefore, better distribution of guidelines could help to support more appropriate DI ordering.

Czembirek et al. (2002) suggest that guidelines alone will not impact DI ordering practices; however, education and training could promote more appropriate DI ordering (Bautista et al. 2009; Czembirek et al. 2002; Dillon and Slanetz 2010; Logie et al. 2010). Education and training could be delivered in many ways including having guidelines "available at work stations, dedicating conferences to specifically address imaging decision support and introducing new residents" (Logie et al. 2010: 254) to guidelines early in their training. Thus, current literature suggests that CDS systems, education and guidelines could help to promote appropriate DI ordering. 


\section{Factors Contributing to Inappropriate Ordering}

To understand potential solutions, examining factors that contribute to inappropriate DI ordering is beneficial. Specifically, legal liability, patient demand and guideline familiarity were all noted as contributing factors to inappropriate DI ordering in the current literature. Researchers suggest that legal liability contributes to inappropriate DI ordering (Butler and Stolberg 2004; DeCampo et al. 2006; Dillon and Slanetz 2010; HCC 2010). Meanwhile, physicians may succumb to pressure from patients to order DI in unnecessary circumstances (Dillon and Slanetz 2010). However, the extent of patient demand as a contributing factor to inappropriate DI ordering is unknown. Last, physicians may not be familiar with their jurisdiction's guidelines (Bautista et al. 2009; DeCampo et al. 2006); this could be a reflection of their current formats. For example, in the United States, the American College of Radiology (ACR) manages Appropriateness Criteria for physicians to reference. It is available in several formats: booklet, PDF, CD-ROM, webpage and file to download to a personal digital assistant (PDA; Sistrom 2005). In a survey of 126 physicians, only $2.4 \%$ used the ACR Appropriateness Criteria (Bautista et al. 2009). Therefore, a more seamless approach to incorporating guidelines into physicians' workflow could prove beneficial - perhaps through a CDS system. Understanding the underlying causes of inappropriate ordering could inform ways to minimize its prevalence. Overall, legal liability, patient demand and guideline familiarity were present in the literature as contributing factors to inappropriate DI ordering.

\section{Consequences to Inappropriate DI Ordering}

Inappropriate DI ordering raises concerns for patient safety (Brenner and Hall 2007) and places strains on human (HCC 2010) and financial resources (CAR 2010), warranting the need to investigate solutions to this phenomenon. Certain DI modalities (e.g., radiography, CT and PET) expose patients to radiation. While the clinical benefits often outweigh the radiation exposure risks, CT examinations could be replaced with MRI or sonography when applicable, and these do not expose a patient to radiation (CAR 2005b). Although the "lifetime cancer risk estimate associated with an abdominal CT scan on a 25 year old is $0.05 \%$ which is 1 in 2000" (Brenner 2010: 66), which may seem small, the escalating number of CT examinations is concerning on a population level. In fact, many researchers note patient safety as the primary concern surrounding inappropriate DI ordering (Bautista et al. 2009; Butler and Stolberg 2004; Czembirek et al. 2002; Schueler 2008; Sistrom 2005).

Inappropriate DI ordering also raises concerns about limited financial and human resources. In the 2005-2006 fiscal year, approximately $\$ 2.2$ billion were spent on DI services in Canadian hospitals (CIHI 2008). Minimizing inappropriate DI ordering could result in financial savings to the healthcare system. Last, CAR "remains concerned about the increasing workload and staffing levels needed to keep pace with the growth in the number of scanners" (HCC 2010). Overall, these consequences to inappropriate DI ordering warrant examining solutions to mitigate this phenomenon.

\section{"In the 2005-2006 fiscal year, approximately $\$ 2.2$ billion were spent on DI services in Canadian hospitals."}

\section{Methods}

This research used a qualitative approach in an effort to gain a rich understanding of physician participants' experiences and processes of ordering DI. More specifically, grounded theory was applied as a means of generating theory from the data (Jackson and Verberg 2007).

\section{Participants and Setting}

English-speaking non-radiologist physicians who were able to order DI services in their Canadian jurisdiction were invited to participate in this study. Radiologists were excluded because of their expected greater knowledge of the field of radiology in comparison to non-radiologist physicians.

All interviews were conducted via telephone as participants were from various locations across Canada.

\section{Data Collection}

The interviews were semi-structured to allow for some flexibility while also ensuring the most relevant topics were discussed. All interviews were recorded, with permission from the participants. After the interviews, the researcher transcribed and reviewed all audio recordings. The interviews included a short demographic questionnaire followed by interview questions (approximately 30 minutes in total). Although the lengths of qualitative interviews can vary, they are typically between 30 minutes and a few hours (Banister et al. 2011). According to Bowling (2009), Jackson and Verberg (2007), Richards (2005), Strauss and Corbin (1998), qualitative interview data should be collected until saturation is achieved (i.e., when new participants do not contribute any new information but, rather, repeat and confirm information discussed by previous participants). Data were collected in this study until saturation occurred (Bowling 2009; Jackson and Verberg 2007; Richards 2005; Strauss and Corbin 1998).

\section{Data Analysis}

Using grounded theory, data were analyzed using the constant comparative method throughout the data collection process. In this method, "data collection and analysis are interrelated processes" (Corbin and Strauss 1990: 6). Thus, data analysis is a continuous process that begins during data collection. 
TABLE 1.

Demographic questionnaire results

\begin{tabular}{l|l} 
Physician Demographics & Findings \\
Physician experience & $9 / 12 ; 75 \%$ over 15 years' experience \\
Education & $10 / 12 ; 83 \%$ educated in Canada and UK \\
Specialty & General practice and various specialties \\
Requisitions/week & 23.58 (mean) \\
Non-challenging DI Orders & $48.83 \%$ \\
Imaging modalities & Radiography/CT/sonography/MRI/PET \\
CT = computed tomography; DI = diagnostic imaging; MRI = magnetic resonance imaging; $P E T$ = positron emission tomography; UK = United Kingdom.
\end{tabular}

First, concept formation occurred by using three levels of coding. In the first level of coding, patterns found in the interview transcripts were coded in order to identify key words or themes. Next, level one codes were compared in an effort to condense similar words or themes into categories. Finally, level three coding involved recognizing core variables; a core variable is a conceptual element that "focuses the theory and accounts for most of the variation in a pattern of behaviour that is both relevant and problematic for the participants involved" (Jackson and Verberg 2007: 175). After this, concept development occurred, which required reduction. Here, the researcher compared the categories and identified relationships that could be characterized under a more unifying category. Concept formation also involved selective sampling of the literature and data to support the core variables that emerged. Last, theoretical coding was performed, whereby unused concepts were reintroduced into the theoretical findings and compared (Jackson and Verberg 2007). In summary, grounded theory was used to generate theory from the data.

\section{Results}

In this section, results from the key informant interviews are highlighted. After presenting results from the demographic questionnaire, appropriate and inappropriate ordering are discussed - the major themes that emerged from the interviews.

\section{Demographic Data}

Twelve English-speaking non-radiologist physicians who were able to order DI services in their Canadian jurisdiction participated in this study: four general practitioners, two neurologists, two geriatricians, one rheumatologist, one respirologist, one hepatologist and one emergency physician. The experience of physicians ranged from 4.5 to 35 years, with a mean of 20.33 years. Most participants completed their medical education in Canada (58\%) and the United Kingdom (25\%).

Before commencing the interviews, participants were also asked to provide some basic information about their DI ordering practices. This included asking the participants to recall how many DI orders they would typically place in a week. Answers ranged from 1.5 to 85 , with a mean of 23.58. Participants were also asked to ascribe a percentage to how many DI requisitions placed in a week were routine (i.e., a non-challenging clinical scenario). Answers ranged from 0 to $80 \%$ being non-challenging requisitions, with a mean of $48.83 \%$. Last, participants were asked to identify the imaging modalities that they most typically used. The majority of participants listed using more than one modality. Participants described using radiography $(9 / 12$; $75 \%)$, CT (9/12; 75\%), sonography (7/12; 58\%), MRI (6/12; $50 \%)$ and PET $(1 / 12 ; 8 \%)$ most typically. These findings are represented in Table 1.

\section{Interview Responses}

The following themes emerged from the key informant interviews. Appropriate and inappropriate ordering emerged as the overarching themes of this study. There were several subthemes, including what methods of information support physicians use to guide challenging DI decisions, and solutions and contributing factors to inappropriate DI ordering.

\section{Information Support Data}

During the interviews, participants noted their top methods of information used during challenging clinical scenarios. These included consulting a radiologist or colleague/specialist (7/11; $64 \%$ ), or searching the literature through UpToDate or Google Scholar $(4 / 11 ; 36 \%)$.

\section{Methods for Supporting More Appropriate Ordering}

During the interviews, participants were prompted to answer whether they thought CDS systems, guidelines or education would help to reduce inappropriate DI ordering. Interestingly, using CDS systems as a method to reduce inappropriate ordering was met with mixed responses. Four participants (4/9; $44 \%$ ) were undecided as to whether CDS systems could reduce inappropriate DI ordering, as is illustrated in the following interview excerpt:

"Certainly, decision support is a complex thing ...

I'm not entirely sure it would [reduce inappropriate 
ordering]. Now, it can do a whole host of other things, but, I'll sit on the fence on that." (Participant 6)

Three participants $(3 / 9 ; 33 \%)$ thought CDS systems could minimize inappropriate DI ordering, whereas two participants $(2 / 9 ; 22 \%)$ did not agree. Overall, using CDS systems as a means to reduce inappropriate DI ordering was met with mixed responses. Meanwhile, the majority of participants thought that education could $(8 / 10 ; 80 \%)$ and guidelines could not (7/9; $78 \%)$ reduce inappropriate DI ordering.

Without prompting from the interviewer, a quarter of participants $(3 / 12 ; 25 \%)$ mentioned how stronger communication on DI requisitions (such as a free-text field to an electronic order entry system) could help to promote more appropriate ordering. Similarly, some participants $(3 / 12 ; 25 \%)$ also described how restricting ordering authority for select DI examinations to certain physician specialties could also support more appropriate DI ordering.

\section{Factors That Influence Inappropriate DI Ordering}

Participants were also asked to describe factors that they believed contribute to inappropriate DI ordering. Interestingly, 10/11 participants $(91 \%)$ commented on how patient demand contributes to the problem. Particularly, participants described how patients sometimes receive unreliable information from the media or from family and friends and then present this information to their physician. Similarly, participants noted how physicians might order a DI examination to alleviate a patient's anxiety. Lastly, participants mentioned how physicians might succumb to patient demand because they fear an unsatisfied patient will leave their care to seek the advice of another physician:

"A lot of people look-up and Google things ... and 95\% of the things people look up I would say are garbage. The Internet is a very dangerous place." (Participant 10)

\section{Interestingly, 10/11 participants (91\%) commented on how patient demand contributes to the problem.}

Overall, patient demand was mentioned by the majority of participants as influencing DI ordering practices.

Next, participants mentioned how legal liability and duplicate ordering contribute to inappropriate DI ordering. Specifically, half of the participants $(6 / 12 ; 50 \%)$ described how the fear of litigation might influence physicians to order DI unnecessarily:

"Even though I know that the likelihood of the test being abnormal is low, I feel that it is necessary to actually show that, in case of the rare situation where it wasn't. So, I would say, medico-legally, that's in the back of my mind." (Participant 2)

This contrasts the opinion of one participant, who felt protected by the Canadian Medical Protective Association.

Lastly, participants were asked how they determine if a DI examination has already been performed or is scheduled to take place. Interestingly, the majority $(8 / 12 ; 67 \%)$ of participants noted that they would ask the patient directly:

"The most reliable [way to know if a DI examination has already been performed or scheduled to take place] is probably still asking the patient." (Participant 7)

Meanwhile, the remainder of participants would consult their picture archiving and communication system (PACS) or other electronic system.

\section{Discussion}

Overall, appropriate and inappropriate ordering emerged as the overarching themes of this study. Subthemes to appropriate ordering included the thought processes of physicians and the information support physicians would seek during challenging clinical scenarios. Other subthemes included contributing factors (such as patient demand, legal liability and duplicate ordering) and solutions (including CDS systems, guidelines, education, requisition communication and restrictions to ordering authority) to inappropriate DI ordering. These findings were then compared with the current literature to discover previous findings on this subject.

\section{Methods for Supporting Appropriate Ordering}

During the key informant interviews, participants were asked to comment on possible solutions to reduce inappropriate ordering. Participant responses were mixed with respect to CDS systems. Specifically, when asked to indicate whether participants thought CDS systems could help to promote more appropriate ordering, four $(4 / 9 ; 44 \%)$ were undecided, three (3/9: $33 \%)$ thought they could and two $(2 / 9 ; 22 \%)$ did not think they could. Generally, this question explored participant impressions on whether CDS systems could minimize inappropriate ordering. Although not referring to CDS systems or healthcare specifically, research suggests that "perceived usefulness had a very strong positive effect on intention to use information technology" (Venkatesh et al. 2002: 311). Therefore, human factors (such as how physicians' attitudes may affect acceptance of a technology) are important to examine when introducing a new technology, such as a CDS system, into the healthcare setting. Meanwhile, research on physician perceptions of CDS systems could be conducted with more focused questions to determine user needs and acceptance before the design, development and implementation of such a system. 
Again, the majority of participants (7/9; 78\%) did not believe guidelines would help to reduce inappropriate DI ordering. This attitude was reflected in responses to the interview question that asked participants to disclose their top methods of information support used in challenging clinical scenarios. In this question, none of the participants responded that guidelines were a means of information support. In another study, only two physician participants $(2 / 126 ; 1.59 \%)$ claimed they would use guidelines (the ACR's Appropriateness Criteria) as their top method of obtaining information support (Bautista et al. 2009). Thus, the interview responses are in line with the current literature available on the topic of guideline use for DI. However, if guidelines are to be used as the basis for a CDS system, differing formats need to be evaluated.

Interestingly, two participants who did not believe that guidelines could reduce inappropriate DI ordering commented that they felt burdened with guidelines from many sources, not just those related to DI. This could be a comment on the current format of DI guidelines. Another researcher noted that guidelines need to be seamlessly incorporated into a physician's workflow for optimal adoption (Czembirek et al. 2002). In fact, CAR recognizes that its Diagnostic Imaging Referral Guidelines (2005a) (which are available in a booklet or PDF) could be incorporated into a CDS system for greater adoption (CAR 2009). Perhaps evaluating different formats of guidelines, such as incorporating guidelines into CDS systems, would be informative.

The majority of participants $(8 / 10 ; 80 \%)$ believed that education (including medical school education and continuing medical education) could reduce inappropriate DI ordering. However, presence of this finding in the literature is largely anecdotal. One study noted that only $21.4 \%$ of medical schools in the United States require radiology clerkships (Dillon and Slanetz 2010), presenting few opportunities to research how these clerkships affect DI ordering practices. Another study investigated how a lecture-based session followed by group exercises (based on the ACR Appropriateness Criteria) challenged medical student participants to think in a different way regarding radiology. In fact, close to $90 \%$ of these medical school participants reported gaining "a solid understanding of the indications for the use of various imaging tests for specific common clinical scenarios" (Dillon and Slanetz 2010: 915). Overall, research in this area is limited; however, interest in education to promote more appropriate ordering is present, warranting more research.

Some participants $(3 / 12 ; 25 \%)$ suggested that improved communication on DI requisitions could promote more appropriate ordering practices. A study by Cohen et al. supports this finding, expressing that "an accurate history is essential if the radiologist is to be able to identify and answer the specific clinical questions that the referring clinician needs answered"
(2006: 239). However, they found in their study, which investigated the quality of radiography requisitions in the intensive care unit, that in $24 \%$ of cases, the information that was provided was either insufficient or incomplete (Cohen et al. 2006). This is further supported in a study surveying radiologists, whereby $72 \%$ of participants "reported that they frequently needed more clinical information about their patients than received" (Boonn and Langlotz 2009: 359). Therefore, focusing on how communication could be improved on DI requisitions may support more appropriate DI ordering. For example, having a free-text box on an electronic requisition as part of a CDS system could help to ensure that all necessary information is provided.

Restricting ordering authority was also identified by several participants $(3 / 12 ; 25 \%)$ as a possible solution to inappropriate DI ordering. Participants described restricting ordering authority by requiring approval from a radiologist, or by allowing only select physician specialties to order certain DI examinations. In fact, in Canada MRI and CT examinations were previously available for only specialist physicians to order; however, this ordering authority has changed, granting general practitioners ordering privileges for these modalities given certain clinical indications in specific Canadian jurisdictions (HCC 2010). Examining jurisdictions that have restrictions to accessing certain DI modalities is necessary to determine the effectiveness of this step as a means to reducing inappropriate ordering. Restricting ordering authority could be done in a CDS system, whereby physician identification numbers correspond with ordering restrictions. Further research is needed in this area.

\section{Factors That Influence Inappropriate Ordering}

Through the interviews, participants discussed several factors that influence inappropriate ordering: patient demand, legal liability and duplicate ordering. Again, the majority of participants $(10 / 11 ; 91 \%)$ described how patient demand might influence how a physician orders DI services. According to a Statistics Canada report, more than one third of adults in Canada use the Internet to search for health information, and a third of these present their findings to their physician (Underhill and McKeown 2008). The discussion of patient demand in the literature is largely anecdotal. In the absence of DI-related literature, parallels can be drawn to other areas in healthcare such as prescription medication ordering. For example, results from another study found that "nearly half of all incidents [studied] of patient pressure to prescribe resulted in the patient being prescribed the medication they requested" (Lewis and Tully 2011: 7). Interestingly, the prescribing physicians deemed the order to be inappropriate in two thirds of the cases (Lewis and Tully 2011). In another study, almost $14 \%$ of patients who were denied a medication that they requested switched physicians (Lee and Begley 2011). This exemplifies the interview finding 
that physicians might submit to patient requests for fear of the patient leaving to seek the advice of another physician. Overall, more research is necessary to further understand the extent of patient demand on inappropriate ordering with respect to DI.

Legal liability was mentioned in half of the interviews as a contributing factor to inappropriate DI ordering. The discussion of legal liability in the literature is mostly anecdotal. As with patient demand, more research is necessary to determine the influence of legal liability on DI ordering practices.

Two thirds of participants ask the patient directly to determine whether an examination has already been performed or is scheduled to take place. However, another study emphasized that patients may not accurately recall their DI histories (Baumann et al. 2011). Particularly, patient participants who presented to an emergency department with acute abdominal pain were asked to recall whether they had received a CT examination in the previous five years. Interestingly, $31 \%$ of these patients claimed they had not. However, by referencing the hospital's electronic health record, researchers discovered that $39 \%$ of participants who made this claim had indeed received a CT examination (Baumann et al. 2011). Thus, depending on patients to recall their DI history - as per the majority of participants in the current study - may not be a dependable method.

Overall, results from the key informant interviews were compared with the current literature. Methods for supporting more appropriate ordering included introducing CDS systems, guidelines, education, requisition communication and restricting ordering authority. Results relating to factors that influence inappropriate ordering were also compared to the available literature.

\section{Limitations}

As physician recruitment rates were low (4.5\%), only radiologists were excluded from the eligibility criteria; perspectives from one specialty may not represent those of other specialties. To the authors' knowledge, there is no publicly available source listing the total number of DI referrals broken down by specialty in Canada; thus, the ordering practices of participants in this study cannot be compared against data from all referring physicians in Canada to determine if this group is representative of DI ordering practices of the population. Also, physician participants were given a brief definition of a CDS system. However, this may not have equipped participants sufficiently to answer whether they believed a CDS system could reduce inappropriate DI ordering.

\section{Contributions to Health Informatics Practice}

This study formed the basis for the design of a DI CDS system prototype (see Griffith et al. 2013). The prototype is purely visual and is designed to communicate the features that are important to include in a working system and to elicit feedback from future users of the system. Features of this prototype design include a free-text box to improve requisition communication between the referring physician and radiologist. As well, to promote patient safety, an alert appears when a physician orders an examination that has already been performed or is scheduled. A screen capture of this feature is shown in Figure 1.

Another feature includes having a cumulative radiation dose history available in order for physicians to make more informed decisions.

Lastly, this prototype design includes links to directly access

\section{Depending on patients to recall their DI history - as per the majority of participants in the current study - may not be a dependable method.}

the most commonly reported methods of obtaining information support. Thus, findings from the key informant interviews and a literature review formed the basis for a DI CDS system prototype design. Future directions will include testing this prototype for usability.

\section{Conclusion}

As up to $30 \%$ of DI examinations could be inappropriate, it is critical to examine the solutions and contributing factors to inappropriate ordering. This is especially important with the recent increases in DI use. Using grounded theory, appropriate and inappropriate DI ordering emerged as the overarching themes of this study. This included several subthemes: methods of information support sought by physicians, solutions to inappropriate ordering and factors that contribute to inappropriate DI ordering. Contributing to the field of health informatics, this study was interested in the physician perspective of DI ordering practices and technology to mitigate inappropriate DI ordering (such as CDS systems) - an angle that is currently absent from DI-related literature. Promoting more appropriate ordering could improve patient safety and the responsible use of limited resources. HQ

\section{References}

Atlantic Health Sciences Centre, Canadian Association of Radiologists and Medicalis. 2005. Demand-Side Control of Diagnostic Imaging through Electronic Clinical Decision Supports: A Pilot Using Appropriateness Guidelines. Ottawa, ON: Canadian Association of Radiologists. Retrieved October 6, 2010. <http://www.car.ca/uploads/ nb_approp_051005_finaldraft.pdf >.

Banister, P., G. Bunn, E. Burman, J. Daniels, P. Duckett, D. Goodley et al. 2011. Qualitative Methods in Psychology: A Research Guide (2nd ed.). Berkshire, United Kingdom: Open University Press.

Baumann, B., E. Chen, A. Mills, L. Glaspey, N. Thompson, M. Jones and M. Farner. 2011. "Patient perceptions of computed tomographic imaging and their understanding of radiation risk and exposure." Annals of Emergency Medicine 58(1): 1-7. 
FIGURE 1.

Screen capture of duplicate order alert in a clinical decision support system prototype

Smith, John 45627281

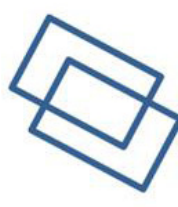

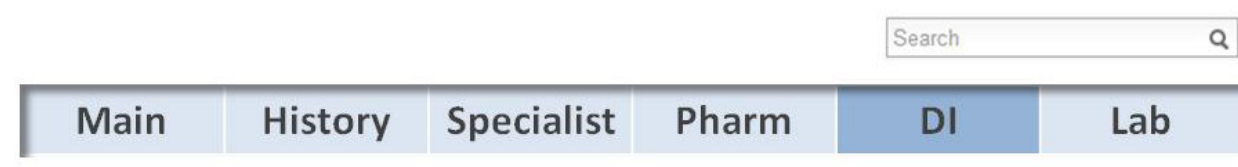

Symptoms

Selected Symptoms

\begin{tabular}{|l|lr|}
\hline PACS & Possible Duplicate Order \\
\hline Order & Non-Urgent MRI booked for May 25, 2 \\
\hline CAR Guidelines & Requested by: Dr. Patricia Walter (Neu \\
\hline Information Supr & Radiologist Approval: Dr. Samantha Re \\
\hline Radiation Exposi & & Cance \\
\hline Notes & Headache & $\square$ \\
& Impaired Colour Vision & $\square$ \\
& Impaired Judgement & \\
\hline
\end{tabular}

Continue

CAR = Canadian Association of Radiologists; $\mathrm{DI}$ = diagnostic imaging; $\mathrm{MRI}$ = magnetic resonance imaging; $\mathrm{PACS}=$ picture archiving and communication system .

Bautista, A., J. Amorosa, A. Burgos, B. Nickel, A. Tilara and J. Yoon. 2009. "Do Clinicians Use the American College of Radiology Appropriateness Criteria in the Management of Their Patients?" American Journal of Roentgenology 192: 1581-85.

Boonn, W. and C. Langlotz. 2009. "Radiologist Use of and Perceived Need for Patient Data Access." Journal of Digital Imaging 22(4): 357-62.

Bowling, A. 2009. Research Methods in Health (3rd ed.). New York: McGraw Hill.

Brenner, D. 2010. "Should we be concerned about the rapid increase in CT usage?" Reviews on Environmental Health 25(1): 63-67.

Brenner, D. and E. Hall. 2007. "Computed Tomography - An Increasing Source of Radiation Exposure." New England Journal of Medicine 357: 2277-84.

Butler, G. and H. Stolberg. 2004. "The Use of Cross-Sectional Diagnostic Imaging in a Major Canadian Health Care Centre." Canadian Association of Radiologists Journal 55(3): 174-77.

Canadian Association of Radiologists. 2005a. Diagnostic Imaging Referral Guidelines: A Guide for Physicians (1st ed.). Saint Laurent, QC: Author < Canadian Association of Radiologists. 2005b. Do You Need That Scan? Ottawa, ON: Author. Retrieved June 10, 2012. <http://car. ca/uploads/news\%20publications/car_cat_scan_eng.pdf>.
Canadian Association of Radiologists. 2007. Manitoba Project on Diagnostic Imaging Demand-Side Control. Ottawa, ON: Author. Retrieved May 20, 2014. < http://www.car.ca/uploads/about/ manitoba\%20i\%20project_final\%20report.pdf >.

Canadian Association of Radiologists. 2009. Appropriateness Projects. Ottawa, ON: Author. Retrieved June 2, 2012. <http://www.car.ca/en/ about/reports/appropriateness.aspx>.

Canadian Association of Radiologists. 2010. As Many as 30\% of Diagnostic Imaging Procedures Deemed Unnecessary: Canadian Association of Radiologists Helps Physicians Choose Best Test First [News Release]. Ottawa, ON: Author. Retrieved November 3, 2010. <http://www.car. ca/uploads/news\%20publications/car_news_release_20100927.pdf $>$.

Canadian Institute for Health Information. 2008. Medical Imaging in Canada 2007. Ottawa, ON: Author. Retrieved May 20, 2014. $<$ https://secure.cihi.ca/free_products/MIT_2007_e.pdf>.

Canadian Institute for Health Information. 2013. Medical Imaging: Medical Imaging Technology 2012 Data Release - Static Figures \& Tables. Ottawa, ON: Author. Retrieved May 20, 2014. < http://www. cihi.ca/CIHI-ext portal/internet/en/tabbedcontent/types+of+care/ specialized+services/medical+imaging/cihi010642\#_Reports_and_ Analyses>.

Cohen, M., S. Curtin and R. Lee. 2006. "Evaluation of Radiology Requisitions for Intensive Care Unit Patients." Academic Radiology 13(2): 236-40. 
Corbin, J. and A. Strauss. 1990. "Grounded Theory Research: Procedures, Canons, and Evaluative Criteria." Qualitative Sociology 13(1): 3-21.

Czembirek, H., F. Fruhwald, H. Imholf, F. Kainberger and P. Pokieser. 2002. "Guidelines and Algorithms: Strategies for Standardization of Referral Criteria in Diagnostic Radiology." European Radiology 12: 673-79.

DeCampo, J., K. Lau and T. Phan. 2006. "Stratification of Radiological Test Ordering: Its Usefulness in Reducing Unnecessary Tests with Consequential Reduction in Costs." Australasian Radiology 50: 335-38.

Dillon, J. and P. Slanetz. 2010. "Teaching Evidence-Based Imaging in the Radiology Clerkship Using the ACR Appropriateness Criteria." Academic Radiology 17(7): 912-16.

Government of Saskatchewan, Department of Health. 2007. Diagnostic Imaging Projects: Decision Support Tools. Regina, SK: Author. Retrieved November 14, 2010. <http://www.health.gov.sk.ca/diagnosticimaging-decision-support-tool>.

Government of Western Australia, Department of Health. 2007. Imaging Pathways. Perth, Western Australia. Retrieved April 8, 2011. <http://www.imagingpathways.health.wa.gov.au/includes/dipmenu/>.

Griffith, J., E. Borycki and A. Kushniruk. 2013. "Tailoring Decision Support to Suit User Needs: A Diagnostic Imaging Example.” Studies in Health Technology and Informatics 183: 132-36.

Health Council of Canada. 2010. Decisions, Decisions: Family Doctors as Gatekeepers to Prescription Drugs and Diagnostic Imaging in Canada. Ottawa, ON: Author. Retrieved October 14, 2010. <http://healthcouncilcanada.ca/docs/rpts/2010/HSU/DecisionsHSU_Sept2010. pdf $>$.

Jackson, W. and N. Verberg. 2007. Methods: Doing Social Research (4th ed.). Toronto, ON: Pearson.

Kahn, C. and D. Tjahjono. 1999. "Promoting the Online Use of Radiology Appropriateness Criteria." Radiographics 19(6): 1673-81.

Lee, D. and C. Begley. 2011. "Physician Switching After Drug Request Refusal." Health Marketing Quarterly 28(4): 304-16.

Lewis, P. and M. Tully. 2011. "The Discomfort Caused by Patient Pressure on the Prescribing Decisions of Hospital Prescribers." Research in Social and Administrative Pharmacy: RSAP 7(1): 4-15.

Logie, C., S. Smith and P. Nagy. 2010. "Evaluation of Resident Familiarity and Utilization of the ACR Musculoskeletal Study Appropriateness Criteria in the Context of Medical Decision Support." Academic Radiology 17(2): 251-54.

Massachusetts General Hospital, Department of Radiology. n.d. Radiology Order Entry and Decision Support. Boston, MA: Author. Retrieved October 14, 2010. <http://www.massgeneralimaging.org/ roel>.
Musen, M., Y. Shahar and E. Shortliffe. 2006. "Clinical Decision Support Systems." In E. Shortliffe and J. Cimino, eds., Biomedical Informatics: Computer Applications in Health Care and Biomedicine. New York, NY: Springer Science and Business Media.

Ontario Ministry of Health and Long-Term Care, Diagnostic Imaging. 2009. MRI/CT Decision Support Tool for Referring Physicians. Toronto, ON: Author. Retrieved January 12, 2011. <https://www.mrictdecisionsupporttool.ca/OEBI/IndicationSearch/Help_Introduction>.

Richards, L. 2005. Handling Qualitative Data. London: Sage Publishing.

Rosenthal, D., J. Weilburg, T. Schultz, J. Miller, V. Nixon, K. Dreyer and J. Thrall. 2006. "Radiology order entry with decision support: Initial clinical experience." Journal of American College of Radiology 3: 799-806.

Schueler, B. 2008. "Incorporating Radiation Dose Assessments into the ACR Appropriateness Criteria." Journal of the American College of Radiology 5(6): 775-76.

Sistrom, C. 2005. "The ACR Appropriateness Criteria." American College of Radiology 2(1): 61-67.

Sistrom, C. and J. Honeyman. 2002. "Relational Data Model for the American College of Radiology Appropriateness Criteria." Journal of Digital Imaging 15(4): 216-25.

Strauss, A. and J. Corbin. 1998. Basics of Qualitative Research (2nd ed.). Thousand Oaks, CA: Sage Publications.

Underhill, C. and L. McKeown. 2008. "Getting a Second Opinion: Health Information and the Internet." Health Reports 19(1): 65-69.

Venkatesh, V., C. Speier and M.G. Morris. 2002. "User Acceptance Enablers in Individual Decision Making About Technology: Toward an Integrated Model.” Decision Sciences 33(2): 297-316.

\section{About the Authors}

Janessa K. Griffith, BA, MSc, is a research coordinator with the School of Health Information Science at the University of Victoria, in Victoria, British Columbia.

Elizabeth M. Borycki, RN, PhD, is an associate professor at the School of Health Information Science, University of Victoria.

Andre W. Kushniruk, PhD, is a professor at the School of Health Information Science, University of Victoria. 